\title{
Platelet-to-lymphocyte ratio is an independent predictor of chemoradiotherapy-related esophageal fistula in esophageal cancer patients
}

\author{
Dong Han", Jiajia Zhang", Jingjing Zhao, Tongda Lei, Xi Chen, Tian Zhang, Hui Wei, Yong Guan, \\ Jing Wang, Wencheng Zhang, Lujun Zhao, Jun Wang, Zhiyong Yuan, Yongchun Song, Ningbo Liu, \\ Qingsong Pang, Ping Wang^
}

Department of Radiation Oncology, Tianjin Medical University Cancer Institute \& Hospital, National Clinical Research Center for Cancer, Key Laboratory of Cancer Prevention and Therapy, Tianjin's Clinical Research Center for Cancer, Tianjin, China

Contributions: (I) Conception and design: Q Pang, W Zhang, D Han; (II) Administrative support: P Wang, Q Pang, L Zhao, J Wang, Z Yuan, Y Song, N Liu; (III) Provision of study materials or patients: J Zhao, X Chen, J Zhang, T Lei, T Zhang, Y Guan; (IV) Collection and assembly of data: D Han, J Zhang, T Lei, H Wei, J Wang; (V) Data analysis and interpretation: D Han, J Zhang; (VI) Manuscript writing: All authors; (VII) Final approval of manuscript: All authors.

\#These authors contributed equally to this work.

Correspondence to: Ping Wang. Department of Radiation Oncology, Tianjin Medical University Cancer Institute \& Hospital, Huanhuxi Road, Hexi District, Tianjin, China. Email: wangping@tjmuch.com.

\begin{abstract}
Background: Neutrophil-to-lymphocyte ratio (NLR), platelet-to-lymphocyte ratio (PLR), and monocyteto-lymphocyte ratio (MLR) are all markers of systemic inflammation response. The role of systemic inflammation in the development of esophageal fistula (EF) has yet to be defined. This study aimed to investigate the predictive value of hematologic measures of inflammation and to set up a predictive model.

Methods: The data of esophageal cancer (EC) patients who received chemoradiotherapy (CRT) in our institution between January, 2015 and January, 2018 were retrospectively collected. The NLR, PLR, and MLR of these enrolled patients were calculated. Univariate and multivariate analyses were performed to find the independent risk factors of EF. Moreover, a nomogram model was developed to predict the probability of fistula occurring in EC patients.

Results: For PLR, the optimal cut-off value was 153. Patients with PLR $>153$ had a higher probability of developing fistula than those with PLR $\leq 153(\mathrm{P}<0.001)$. Multivariate analyses revealed that esophageal stenosis, ulcerative tumor, and PLR were independent factors for EF. Subsequently, a novel nomogram was set up with the C-index of 0.77 to predict the risk of developing EF in EC patients who received CRT.

Conclusions: PLR is an independent predictive indicator for EC patients who receive CRT. These findings will help to facilitate individual risk stratification for the development of EF in patients with EC.
\end{abstract}

Keywords: Esophageal cancer (EC); chemoradiotherapy (CRT); esophageal fistula (EF); risk factors; platelet-tolymphocyte ratio (PLR)

Submitted Apr 09, 2020. Accepted for publication Aug 14, 2020.

doi: 10.21037/atm-20-4053

View this article at: http://dx.doi.org/10.21037/atm-20-4053

^ ORCID: 0000-0002-8470-9644. 


\section{Introduction}

China has the highest risk of esophageal cancer (EC) of any country worldwide, and as the predominant histological type, esophageal squamous cell carcinoma (ESCC) accounts for $90 \%$ of cases nationally (1). Recently, with the development of new strategies to treat human cancer, studies on immunocheckpoint inhibitors have shown encouraging results in ESCC (2). In July 2019, the FDA approved pembrolizumab, the PD-1 inhibitor, for second-line treatment of PD-L1 positive, advanced or metastatic ESCC (3). Currently, immunotherapy has served as the fourth antitumor strategies following surgery, radiotherapy and chemotherapy (2). Increasing clinical trials investigated the combination of immunotherapy and other antitumor strategies (4-9). However, there remains a long way of immunotherapy in ESCC. At present, definitive chemoradiotherapy (CRT) remains the standard treatment for patients with unresectable ESCC without distant metastasis $(10,11)$. Despite CRT having brought about improvements in local control and the long-term survival rates of EC, it has several side effects, of which esophageal fistula $(\mathrm{EF})$ is one of the most serious.

The incidence of EF in EC patients who receive CRT has been reported to be 6-22\% (12-15). The prognosis of EF is abysmal, and the death rate is excessively high. Both tumorrelated and non-tumor-related factors are well known to contribute to EF formation (14). CRT-induced EF is mainly caused by the imbalance between tumor shrinkage and the normal tissue repair system (16-18). Recently, an increasing amount of evidence has demonstrated that inflammation contributes to the development, progression, and metastasis of tumors (19-22). Inflammation can also weaken normal tissue repair (18). Neutrophil-to-lymphocyte ratio (NLR), platelet-to-lymphocyte ratio (PLR), and serum C-reactive protein $(\mathrm{CRP})$ are all associated with systemic inflammatory response $(23,24)$.

Although there have been many reports on EF, only a small number of studies have focused on the association between fistula and inflammation $(14,25)$. However, the evaluation provided by these studies is not comprehensive. Moreover, there was no study about the role of NLR, PLR and MLR in the development of EF, although systemic inflammatory response has been widely used to predict the prognosis of several solid tumors $(23,26-28)$. Therefore, this study was performed to investigate the role of systemic inflammation response in EF development, and to develop a nomogram to predict the risk of $\mathrm{EF}$ in $\mathrm{EC}$ patients who receive CRT.
We present the following article in accordance with the TRIPOD reporting checklist (available at http://dx.doi. org/10.21037/atm-20-4053).

\section{Methods}

\section{Patients and specimens}

The data of EC patients treated in our institute between January 2015 and January 2018 were retrospectively collected. The inclusion criteria were as follows: (I) diagnosed as EC by pathological biopsy; (II) treated with radical CRT or palliative chemoradiation; (III) Karnofsky performance status (KPS) $\geq 70$; and (IV) diagnosed as EF by endoscopy, computed tomography (CT), or X-ray. The exclusion criteria were: (I) previously underwent esophageal surgery or thoracic radiotherapy; (II) the fistula developed before treatment; (III) concomitant with other carcinomas; (IV) suffered from hematopoietic disorders, human immunodeficiency virus, autoimmune disorders, or bone marrow infiltration disorders; or (V) lost to follow-up. Eventually a total of 379 patients were enrolled and analyzed.

Pretreatment peripheral blood sample $(2 \mathrm{~mL})$ for lymphocyte, neutrophil, monocyte and platelet counts was collected using EDTA anticoagulation via venipuncture from enrolled individuals. The Department of Clinical Laboratory in our institution performed the data analyses.

The study was conducted in accordance with the Declaration of Helsinki (as revised in 2013), and was approved by the institutional review board and ethics committee at Tianjin Medical University Cancer Hospital \& Institute (Grant No. bc2020046). The requirement for informed consent was waived because of the retrospective nature of the research.

\section{Data collection}

All data were collected from electronic medical records. Patient information, including demographic, tumor, and treatment-related data, was analyzed. Details of the related parameters are shown in Table 1. Complete blood count (CBC) and CRP were recorded for each patient before treatment, and NLR, PLR, and monocyte-to-lymphocyte ratio (MLR) were calculated. TNM staging followed the UICC-TNM classification 8th edition.

\section{Follow-up}

All patients were evaluated during treatment and 30 days 
Table 1 The characteristics of patients with chemoradiotherapyrelated esophageal fistula

\begin{tabular}{|c|c|c|}
\hline Patient characteristic & Number (\%) & Number of EF (\%) \\
\hline \multicolumn{3}{|l|}{ Age, y } \\
\hline$\leq 60$ & $131(34.6)$ & $18(41.9)$ \\
\hline$>60$ & $248(65.4)$ & $25(58.1)$ \\
\hline Male sex & $313(82.6)$ & $38(88.4)$ \\
\hline \multicolumn{3}{|l|}{ KPS } \\
\hline$\leq 80$ & $116(30.6)$ & $9(20.9)$ \\
\hline$>80$ & $263(69.4)$ & $34(79.1)$ \\
\hline \multicolumn{3}{|l|}{ History of smoking } \\
\hline Yes & $251(66.2)$ & $32(74.4)$ \\
\hline No & $128(33.8)$ & $11(25.6)$ \\
\hline Diabetes & $35(9.2)$ & $5(11.6)$ \\
\hline Esophageal stenosis & $82(21.6)$ & $16(37.2)$ \\
\hline \multicolumn{3}{|l|}{ Tumor location } \\
\hline Cervical/upper & $126(33.3)$ & $13(30.2)$ \\
\hline Middle & $148(39.0)$ & $21(48.8)$ \\
\hline Lower & $105(27.7)$ & $9(21.0)$ \\
\hline Ulcerative tumor & $100(26.4)$ & $22(51.1)$ \\
\hline \multicolumn{3}{|l|}{ T stage } \\
\hline $\mathrm{T} 1-3$ & $309(81.5)$ & $32(74.4)$ \\
\hline $\mathrm{T} 4$ & $70(18.5)$ & $11(25.6)$ \\
\hline \multicolumn{3}{|l|}{$\mathrm{N}$ stage } \\
\hline No-1 & $329(86.8)$ & $37(86.0)$ \\
\hline N2-3 & $50(13.2)$ & $6(14.0)$ \\
\hline \multicolumn{3}{|l|}{ Treatment modality } \\
\hline Concurrent CRT & 265 (69.9) & $20(46.5)$ \\
\hline Sequential CRT & $114(30.1)$ & $23(53.5)$ \\
\hline \multicolumn{3}{|l|}{ Total dose, Gy } \\
\hline$\geq 60$ & $259(68.3)$ & $27(62.8)$ \\
\hline$\geq 50<60$ & $81(21.4)$ & $8(18.6)$ \\
\hline$<50$ & $39(10.3)$ & $8(18.6)$ \\
\hline \multicolumn{3}{|l|}{ NLR } \\
\hline$\leq 2.44$ & $194(51.2)$ & $13(30.2)$ \\
\hline$>2.44$ & $185(48.8)$ & $30(69.8)$ \\
\hline \multicolumn{3}{|l|}{ PLR } \\
\hline$\leq 153$ & $242(63.9)$ & 17 (39.5) \\
\hline$>153$ & $137(36.1)$ & $26(60.5)$ \\
\hline
\end{tabular}

Table 1 (continued)
Table 1 (continued)

\begin{tabular}{|c|c|c|}
\hline Patient characteristic & Number (\%) & Number of EF (\%) \\
\hline \multicolumn{3}{|l|}{ MLR } \\
\hline$\leq 0.30$ & $231(60.9)$ & 15 (34.9) \\
\hline$>0.30$ & $148(36.1)$ & $28(65.1)$ \\
\hline \multicolumn{3}{|l|}{ Alb, g/dL } \\
\hline$\leq 3.5$ & 13 (3.4) & $4(9.3)$ \\
\hline$>3.5$ & $366(96.6)$ & $39(90.7)$ \\
\hline
\end{tabular}

after the therapy was completed. Subsequently, they were followed up every three months for the first year, every six months for the next two years, and annually after that. The assessed examinations consisted of esophagography, CT, cervical lymph nodes, and abdominal ultrasonography.

\section{Variables definition}

EF was defined as a direct connection between the esophagus and adjacent organs. Esophageal stenosis was defined as it being impossible to pass an endoscope through the lesion before treatment. NLR, neutrophil/lymphocyte; PLR, platelet/lymphocyte; MLR, monocyte/lymphocyte.

\section{Statistical analysis}

The occurrence of EF was defined as the final event. Time to EF was calculated from the date of initiation of CRT to the endpoint. Differences between patients who experienced $\mathrm{EF}$ and those who did not were compared by Fisher's exact test and the Mann-Whitney U test. Univariate and multivariate analyses were conducted using logistic regression to estimate the odds ratio (OR). The nomogram for the predictive probability of radiotherapy-related EF was set up with the results of the multivariate analysis. Receiver operator characteristic (ROC) curves were drawn for each inflammatory marker, and the best predictive cut-off values were determined when the Youden Index (sensitivity + specificity - 1) achieves maximum (29). Results from ROC analysis revealed the best cut-off points for NLR, PLR, and MLR in our study as 2.44, 153, and 0.30, respectively. All $\mathrm{P}$ values were reported as two-sided, with an alpha level of $\leq 0.05$. Statistical analyses and statistical 
charts were conducted using the SPSS software package (SPSS, Chicago, IL, USA), R version 3.5 for Windows.

\section{Results}

\section{Patient characteristics}

A total of 379 patients ( 313 males and 66 females) were enrolled in the present study. At diagnosis, the patients had a median age of 64 years (IQR, 58-69 years) old. Of the patients, $9.2 \%$ had diabetes, $21.6 \%$ had esophageal stenosis, and $26.4 \%$ had an ulcerative tumor. In this research, 265 patients received concurrent CRT, while 114 patients received sequential modality. The mean values of NLR, PLR, and MLR were 2.70 $\pm 1.77,148.40 \pm 79.61$, and $0.29 \pm 0.13$, respectively. In our study, the mean serum albumin (Alb) was $4.24 \pm 3.93 \mathrm{~g} / \mathrm{dL}$. The detailed clinical information is shown in Table 1 .

\section{Clinical course of patients with EF}

In our study cohort, 43 patients $(11.35 \%)$ developed EF. Twenty were diagnosed as fistula by CT, 16 were diagnosed by esophagogram, 4 were diagnosed by endoscopic, and 3 were diagnosed by both CT and esophagogram. Among these patients, 6 experienced fistula during treatment, while the remaining 37 patients developed perforation after the CRT had been completed. Radiation/CRT was discontinued for all 6 of the patients who developed fistula during treatment. Of the 37 patients who developed perforation after treatment, the majority $(67.6 \%)$ had been treated with a radiation dose of $\geq 60 \mathrm{~Gy}$, and only 4 patients had received a dose of $<50 \mathrm{~Gy}$. The median interval between the initiation of treatment and the occurrence of fistula was only 3.4 months. The types of EF in our study included esophageal-respiratory fistula (31 patients), esophagealmediastinum fistula (9 patients), and esophagopleural fistula ( 3 patients). The treatment methods for fistula included nutrient canal (53.5\%), parenteral nutrition (18.6\%), gastrostomy (16.3\%), and esophageal stent (11.6\%). At the last follow-up, all of the 43 patients with fistula had died. Of these, 25 patients died from uncontrollable infection, and 18 died from massive hemorrhage.

\section{Risk factors for fistula formation}

In the univariate analysis, esophageal stenosis, ulcerative tumor, NLR, PLR, and MLR were identified as significant factors, while KPS, smoking history, tumor location, $\mathrm{T}$ stage, $\mathrm{N}$ stage, Alb and other factors were not found to be significant (Table 2). The significant parameters were subsequently involved in multivariate analysis. Esophageal stenosis, ulcerative tumors, and PLR were the independent risk factors for the development of fistula (Table 3).

\section{Risk evaluation of fistula}

A nomogram model based on esophageal stenosis, ulcerative tumor, and PLR, combined with age and gender, was established to predict the risk of fistula for EC patients who accepted CRT (Figure 1). By obtaining the points of each involved factor and adding all the variable points, the total risk score was determined, and the risk evaluation of fistula could be directly obtained by the total point axis (30). In the current study, Harrell's C-index for fistula prediction was 0.77. The model had a superior performance in predicting fistula. The calibration curves showed a good agreement between the risk estimation by the nomogram and actual observation (Figure 2).

\section{Discussion}

Our study showed that pretreatment PLR was a potential predictive marker of fistula for EC patients who received CRT. The strong link between fistula and PLR $(\mathrm{P}=0.028)$ could be seen in our study, according to multivariate analysis. Patients with elevated PLR before treatment are more likely to develop fistula compared to those with stable or moderately raised PLR. Since PLR can be calculated from routine, minimally invasive laboratory measurements, it can be easily incorporated into clinical care. These results make pretherapeutic evaluation more workable, which may allow for personalized adjustment of therapeutic strategy (e.g., enhancing nutrition and prompt anti-inflammation).

CRT inevitably has an impact on systemic inflammation. PLR assessed during CRT does not reflect the baseline impact of systemic inflammation on EF in patients with EC; therefore, we evaluated the potential predictive role of PLR before treatment. Our study suggested that PLR is an objective marker for predicting the probability of EF in EC patients.

The potential mechanisms underlying the association of high PLR and EF are poorly understood; thus, we tried to explain this relationship. It has been reported that elevated PLR is indicative of the increased host inflammatory response associated with more aggressive tumor 
Table 2 The univariate analysis of the factors associated with esophageal fistula

\begin{tabular}{|c|c|c|c|}
\hline Variables & OR & $95 \% \mathrm{Cl}$ & $P$ value \\
\hline \multicolumn{4}{|l|}{ Age } \\
\hline$\leq 60$ & 1.0 & & \\
\hline$>60$ & 0.704 & $0.369-1.44$ & 0.285 \\
\hline \multicolumn{4}{|l|}{ Gender } \\
\hline Male & 1.0 & & \\
\hline Female & 0.593 & $0.224-1.569$ & 0.288 \\
\hline \multicolumn{4}{|l|}{ KPS } \\
\hline$\leq 80$ & 1.0 & & \\
\hline$>80$ & 1.765 & $0.818-3.811$ & 0.144 \\
\hline \multicolumn{4}{|c|}{ History of smoking } \\
\hline No & 1.0 & & \\
\hline Yes & 1.554 & $0.756-3.196$ & 0.228 \\
\hline \multicolumn{4}{|l|}{ Diabetes } \\
\hline No & 1.0 & & \\
\hline Yes & 1.342 & $0.491-3.666$ & 0.767 \\
\hline \multicolumn{4}{|c|}{ Esophageal stenosis } \\
\hline No & 1.0 & & \\
\hline Yes & 2.424 & $1.235-4.758$ & 0.008 \\
\hline \multicolumn{4}{|l|}{ Tumor location } \\
\hline Cervical/upper & 1.0 (reference) & & \\
\hline Middle & 1.437 & $0.688-3.002$ & 0.333 \\
\hline Lower & 0.815 & $0.334-1.989$ & 0.653 \\
\hline \multicolumn{4}{|l|}{ Ulcerative tumor } \\
\hline No & 1.0 & & \\
\hline Yes & 3.463 & $3.334-3.608$ & $<0.001$ \\
\hline \multicolumn{4}{|l|}{ T stage } \\
\hline $\mathrm{T} 1-3$ & 1.0 & & \\
\hline $\mathrm{T} 4$ & 1.614 & $0.770-3.384$ & 0.202 \\
\hline \multicolumn{4}{|l|}{$\mathrm{N}$ stage } \\
\hline No-1 & 1.0 & & \\
\hline N2-3 & 1.076 & $0.429-2.698$ & 0.876 \\
\hline \multicolumn{4}{|l|}{ Total dose (Gy) } \\
\hline$\geq 60$ & 1.0 (reference) & & \\
\hline$\geq 50<60$ & 0.942 & $0.410-2.163$ & 0.887 \\
\hline$<50$ & 2.217 & $0.926-5.311$ & 0.074 \\
\hline
\end{tabular}

Table 2 (continued)
Table 2 (continued)

\begin{tabular}{llll}
\hline Variables & OR & $95 \% \mathrm{Cl}$ & $\mathrm{P}$ value \\
\hline NLR & & & \\
$\leq 2.44$ & 1.0 & & \\
$>2.44$ & 2.695 & $1.358-5.347$ & 0.004 \\
PLR & & & \\
$\leq 153$ & 1.0 & & \\
$>153$ & 3.100 & $1.615-5.952$ & $<0.001$ \\
MLR & & & \\
$\leq 0.30$ & 1.0 & & $<0.001$ \\
$>0.30$ & 3.360 & $1.727-6.538$ & $<$ \\
\hline
\end{tabular}

OR, odds ratio; $\mathrm{Cl}$, confidence interval; KPS, Karnofsky performance status; NLR, neutrophil-to-lymphocyte ratio; PLR, platelet-to-lymphocyte ratio; MLR, monocyte-to-lymphocyte ratio.

Table 3 The multivariate analysis of the factors associated with esophageal fistula

\begin{tabular}{|c|c|c|c|}
\hline Variables & OR & $95 \% \mathrm{Cl}$ & $P$ value \\
\hline \multicolumn{4}{|c|}{ Esophageal stenosis } \\
\hline No & 1.0 & & \\
\hline Yes & 2.210 & $1.076-4.539$ & 0.031 \\
\hline \multicolumn{4}{|c|}{ Ulcerative tumor } \\
\hline No & 1.0 & & \\
\hline Yes & 3.378 & $1.701-6.711$ & $<0.001$ \\
\hline \multicolumn{4}{|l|}{ NLR } \\
\hline$\leq 2.44$ & 1.0 & & \\
\hline$>2.44$ & 1.241 & $0.528-2.915$ & 0.621 \\
\hline \multicolumn{4}{|l|}{ PLR } \\
\hline$\leq 153$ & 1.0 & & \\
\hline$>153$ & 2.359 & $1.096-5.080$ & 0.028 \\
\hline \multicolumn{4}{|l|}{ MLR } \\
\hline$\leq 0.30$ & 1.0 & & \\
\hline$>0.30$ & 2.054 & $0.931-4.529$ & 0.075 \\
\hline
\end{tabular}

OR, odds ratio; $\mathrm{Cl}$, confidence interval; NLR, neutrophil-tolymphocyte ratio; PLR, platelet-to-lymphocyte ratio; MLR, monocyte-to-lymphocyte ratio. 


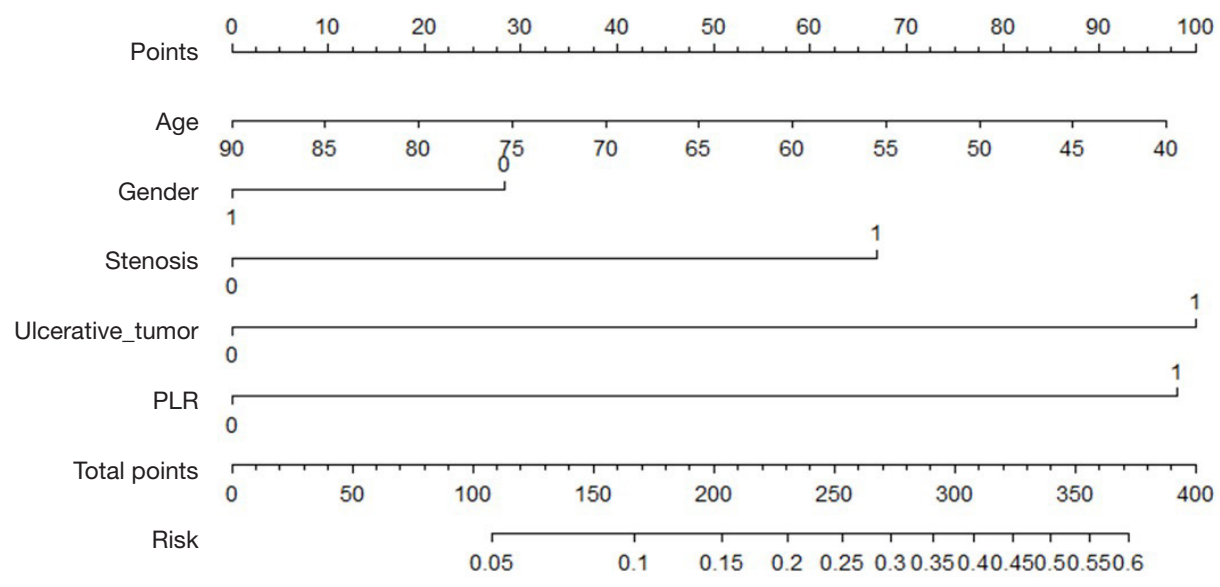

Figure 1 Nomograms for the individualized prediction of radiation-related esophageal fistula in esophageal cancer patients. Gender 0: male, 1: female. Stenosis 0: no esophageal stenosis, 1: esophageal stenosis. Ulcerative-tumor 0: non-ulcerative tumor, 1: ulcerative tumor. PLR 0: PLR $\leq 153,1$ : PLR >153. PLR, platelet-to-lymphocyte ratio.

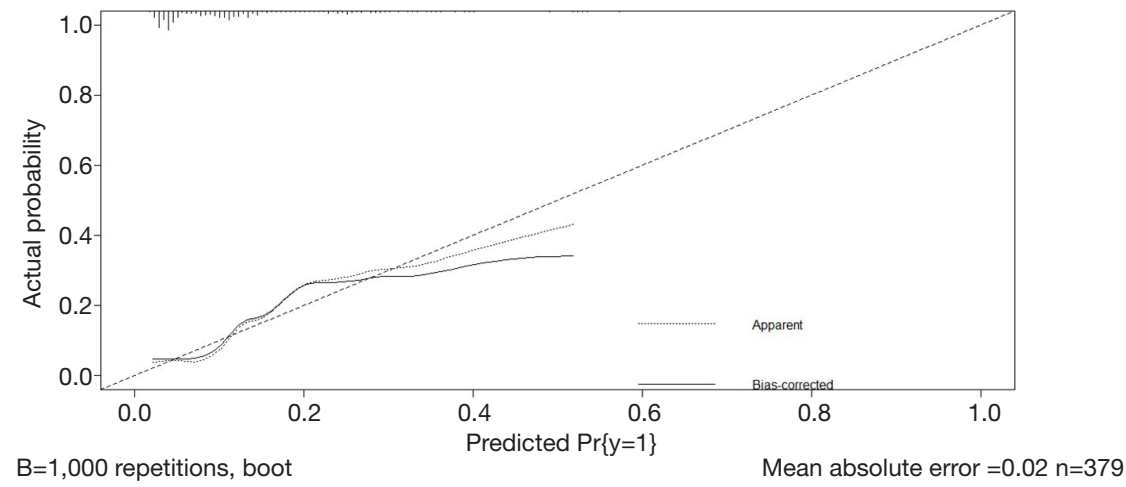

Figure 2 The calibration curve for the test accuracy of the final risk score.

characteristics (31). Inflammatory cytokines and chemokines can be secreted by both tumor and associated host cells (32). Among them, IL6 can stimulate the differentiation of megakaryocytes to platelets $(33,34)$, and it can also stimulate thrombopoietin production and lead to increased platelet counts in patients with cancer (35), which partly results in the elevation of PLR. Also, ulcerative tumors impair the integrity of esophageal mucosa, inducing inflammation. Esophageal stenosis can also cause tissue damage, which is partly attributed to the physical friction of food (36). The above factors elevate the baseline of inflammation, which results in increased production of pro-inflammatory cytokines, preventing tissue repair $(37,38)$. Mirza et al. demonstrated that by blocking the IL-1 $\beta$ pathway, wound healing could be improved in mice, via the downregulation of pro-inflammatory macrophage phenotypes in wounds and the upregulation of pro-healing phenotypes (39). Moreover, the abundance of pro-inflammatory cytokines increases the production of Matrix metalloproteinase (MMP) while reducing the synthesis of tissue inhibitor of metalloproteinase (TIMP); this imbalance further augments degradation of the extracellular matrix, interferes with cell migration, impairs connective tissue deposition, and reduces fibroblast proliferation and collagen synthesis (40). Tissue thereby cannot be promptly repaired when CRT induces tumor shrinkage leaving a thin or even broken wall in the esophagus, and then EF is formed. The fact that these were only partial responses underscores the complexity of fistula development and the influence of multiple factors. Further prospective and preclinical studies are called for to explore the potential mechanisms of fistula development.

It is well known that a nomogram could set up a simple 
diagrammatic representation of a statistical predictive model (30). In the current study, we set up a predictive nomogram model to assess the risk of fistula development in EC patients receiving CRT. This model performed well in predicting fistula by the C-index (0.77). The patients with high scores ( $>340$ points) are 50\% more likely to develop $\mathrm{EF}$ using this model. In consideration of the poor prognosis of EF, clinicians should pay more attention to patients at elevated risk during treatment and follow-up. Appropriate treatment should be given promptly to those patients.

To the best of our knowledge, this is the first study that attempted to explore the role of inflammation in EF comprehensively. Moreover, this is the first report to reveal the predictive role of PLR in EF with a nomogram. However, there are still several limitations to this study. First, it is a retrospective study, so it inevitably has some biases, including selection bias, loss to follow-up, and confounding variables. More prospective studies should be performed to verify our findings. Second, our predictive model was not validated internally or externally; however, internal validation is currently being performed in our institute, and the model will then be externally validated with data from other cooperative hospitals once credible results have been achieved. Third, PLR is one of the surrogates of systemic inflammatory response. While PLR is easy to obtain, it lacks the precision and accuracy of molecular study. Further studies should be conducted to explore the changes in the molecular level via more refined analysis of tissue samples.

\section{Conclusions}

In summary, our study suggested that PLR is an independent predictive indicator of fistula for EC patients who receive CRT. We conclude that 153 may be the best cut-off value for PLR.

\section{Acknowledgments}

We thank all the patients taking part in the study.

Funding: This work is supported by the National Nature Science Foundation of China (Grant No. 81872462, 81773300), and the Clinical Trial Supporting Foundation of Tianjin Medical University Cancer Institute \& Hospital (Grant No. C1707).

\section{Footnotes}

Reporting Checklist: The authors have completed the
TRIPOD reporting checklist. Available at http://dx.doi. org/10.21037/atm-20-4053

Data Sharing Statement: Available at http://dx.doi. org/10.21037/atm-20-4053

Conflicts of Interest: All authors have completed the ICMJE uniform disclosure form (available at http://dx.doi. org/10.21037/atm-20-4053). The authors have no conflicts of interest to declare.

Ethical Statement: The authors is accountable for all aspects of the work in ensuring that questions related to the accuracy or integrity of any part of the work are appropriately investigated and resolved. The study was conducted in accordance with the Declaration of Helsinki (as revised in 2013), and was approved by the institutional review board and ethics committee at Tianjin Medical University Cancer Hospital \& Institute (Grant No. bc2020046). The requirement for informed consent was waived because of the retrospective nature of the research.

Open Access Statement: This is an Open Access article distributed in accordance with the Creative Commons Attribution-NonCommercial-NoDerivs 4.0 International License (CC BY-NC-ND 4.0), which permits the noncommercial replication and distribution of the article with the strict proviso that no changes or edits are made and the original work is properly cited (including links to both the formal publication through the relevant DOI and the license). See: https://creativecommons.org/licenses/by-nc-nd/4.0/.

\section{References}

1. Jemal A, Bray F, Center MM, et al. Global cancer statistics. CA Cancer J Clin 2011;61:69-90.

2. Walsh EM, Kelly RJ. Single agent anti PD-1 inhibitors in esophageal cancer-a first step in a new therapeutic direction. J Thorac Dis 2018;10:1308-13.

3. Barsouk A, Rawla P, Hadjinicolaou AV, et al. Targeted Therapies and Immunotherapies in the Treatment of Esophageal Cancers. Med Sci (Basel) 2019;7:100.

4. Yang H, Wang K, Wang T, Li M, Li B, Li S, et al. The Combination Options and Predictive Biomarkers of PD-1/ PD-L1 Inhibitors in Esophageal Cancer. Front Oncol 2020;10:300.

5. Raufi AG, Almhanna K. Immune checkpoint inhibitors for esophageal cancer: are we moving in the right direction? 
Ann Transl Med 2019;7:S102.

6. Kelly RJ, Smith KN, Anagnostou V, et al. Neoadjuvant nivolumab plus concurrent chemoradiation in stage II/III esophageal/gastroesophageal junction cancer. J Clin Oncol 2019;3:142.

7. Xu N, Yuan X, Wang B, et al. Tislelizumab in combination with chemotherapy for the treatment of Chinese patients (pts) with esophageal squamous cell carcinoma (ESCC): Results from one cohort of an ongoing phase 2 study. J Clin Oncol 2019;37:abstr 14.

8. Zhang B, Qi L, Wang X, et al. Phase 2 study of camrelizumab (anti-PD-1 antibody) combined with apatinib and chemotherapy for the first-line treatment of advanced esophageal squamous cell carcinoma. J Clin Oncol 2019;37:abstr 4033.

9. Lee DH, Kim H, Keam B, et al. Evaluation of safety and tolerability of durvalumab (D) and tremelimumab (T) in combination with first-line chemotherapy in patients (pts) with esophageal squamous-cell carcinoma (ESCC). J Clin Oncol 2019;37:abstr 146.

10. Kato K, Muro K, Minashi K, et al. Phase II study of chemoradiotherapy with 5 -fluorouracil and cisplatin for Stage II-III esophageal squamous cell carcinoma: JCOG trial (JCOG 9906). Int J Radiat Oncol Biol Phys 2011;81:684-90.

11. van Hagen P, Hulshof MC, van Lanschot JJ, et al. Preoperative chemoradiotherapy for esophageal or junctional cancer. N Engl J Med 2012;366:2074-84.

12. Balazs A, Galambos Z, Kupcsulik PK. Characteristics of esophagorespiratory fistulas resulting from esophageal cancers: a single-center study on 243 cases in a 20-year period. World J Surg 2009;33:994-1001.

13. Hihara J, Hamai Y, Emi M, et al. Role of definitive chemoradiotherapy using docetaxel and 5 -fluorouracil in patients with unresectable locally advanced esophageal squamous cell carcinoma: a phase II study. Dis Esophagus 2016;29:1115-20.

14. Tsushima T, Mizusawa J, Sudo K, et al. Risk Factors for Esophageal Fistula Associated With Chemoradiotherapy for Locally Advanced Unresectable Esophageal Cancer: A Supplementary Analysis of JCOG0303. Medicine (Baltimore) 2016;95:e3699.

15. Zhou ZG, Zhen CJ, Bai WW, et al. Salvage radiotherapy in patients with local recurrent esophageal cancer after radical radiochemotherapy. Radiat Oncol 2015;10:54.

16. Han X, Zhao YS, Fang Y, et al. Placement of transnasal drainage catheter and covered esophageal stent for the treatment of perforated esophageal carcinoma with mediastinal abscess. J Surg Oncol 2016;114:725-30.

17. Sivaraman SK, Drummond R. Radiation-induced aortoesophageal fistula: an unusual case of massive upper gastrointestinal bleeding. J Emerg Med 2002;23:175-8.

18. Ohtsu A, Boku N, Muro K, et al. Definitive chemoradiotherapy for T4 and/or M1 lymph node squamous cell carcinoma of the esophagus. J Clin Oncol 1999;17:2915-21.

19. Geng Y, Shao Y, Zhu D, et al. Systemic ImmuneInflammation Index Predicts Prognosis of Patients with Esophageal Squamous Cell Carcinoma: A Propensity Score-matched Analysis. Sci Rep 2016;6:39482.

20. Yodying H, Matsuda A, Miyashita M, et al. Prognostic Significance of Neutrophil-to-Lymphocyte Ratio and Platelet-to-Lymphocyte Ratio in Oncologic Outcomes of Esophageal Cancer: A Systematic Review and Metaanalysis. Ann Surg Oncol 2016;23:646-54.

21. Hu B, Yang XR, Xu Y, et al. Systemic immuneinflammation index predicts prognosis of patients after curative resection for hepatocellular carcinoma. Clin Cancer Res 2014;20:6212-22.

22. Mantovani A, Allavena P, Sica A, et al. Cancer-related inflammation. Nature 2008;454:436-44.

23. Templeton AJ, Ace O, McNamara MG, et al. Prognostic role of platelet to lymphocyte ratio in solid tumors: a systematic review and meta-analysis. Cancer Epidemiol Biomarkers Prev 2014;23:1204-12.

24. Guthrie GJ, Charles KA, Roxburgh CS, et al. The systemic inflammation-based neutrophil-lymphocyte ratio: experience in patients with cancer. Crit Rev Oncol Hematol 2013;88:218-30.

25. Kawakami T, Tsushima T, Omae K, et al. Risk factors for esophageal fistula in thoracic esophageal squamous cell carcinoma invading adjacent organs treated with definitive chemoradiotherapy: a monocentric case-control study. BMC Cancer 2018;18:573.

26. Yang Y, Xu H, Zhou L, et al. Platelet to lymphocyte ratio is a predictive marker of prognosis and therapeutic effect of postoperative chemotherapy in non-metastatic esophageal squamous cell carcinoma. Clin Chim Acta 2018;479:160-5.

27. Chen LC, Li SH, Lo CM, et al. Platelet-to-lymphocyte ratio is an independent prognosticator in patients with esophageal squamous cell carcinoma receiving esophagectomy. J Thorac Dis 2019;11:4583-90.

28. Lin JX, Lin JP, Xie JW, et al. Prognostic importance of the preoperative modified systemic inflammation score for patients with gastric cancer. Gastric Cancer 
2019;22:403-12.

29. Ruopp MD, Perkins NJ, Whitcomb BW, et al. Youden Index and optimal cut-point estimated from observations affected by a lower limit of detection. Biom J 2008;50:419-30.

30. Iasonos A, Schrag D, Raj GV, et al. How to build and interpret a nomogram for cancer prognosis. J Clin Oncol 2008;26:1364-70.

31. Kwon HC, Kim SH, Oh SY, et al. Clinical significance of preoperative neutrophil-lymphocyte versus plateletlymphocyte ratio in patients with operable colorectal cancer. Biomarkers 2012;17:216-22.

32. Balkwill F, Mantovani A. Inflammation and cancer: back to Virchow? Lancet 2001;357:539-45.

33. Lippitz BE. Cytokine patterns in patients with cancer: a systematic review. Lancet Oncol 2013;14:e218-28.

34. Imai T, Koike K, Kubo T, et al. Interleukin-6 supports human megakaryocytic proliferation and differentiation in vitro. Blood 1991;78:1969-74.

35. Klinger MH, Jelkmann W. Role of blood platelets in

Cite this article as: Han D, Zhang J, Zhao J, Lei T, Chen X, Zhang T, Wei H, Guan Y, Wang J, Zhang W, Zhao L, Wang J, Yuan Z, Song Y, Liu N, Pang Q, Wang P. Plateletto-lymphocyte ratio is an independent predictor of chemoradiotherapy-related esophageal fistula in esophageal cancer patients. Ann Transl Med 2020;8(18):1163. doi: 10.21037/atm-20-4053 infection and inflammation. J Interferon Cytokine Res 2002;22:913-22.

36. Zhang $\mathrm{Y}$, Li Z, Zhang $\mathrm{W}$, et al. Risk factors for esophageal fistula in patients with locally advanced esophageal carcinoma receiving chemoradiotherapy. Onco Targets Ther 2018;11:2311-7.

37. Baltzis D, Eleftheriadou I, Veves A. Pathogenesis and treatment of impaired wound healing in diabetes mellitus: new insights. Adv Ther 2014;31:817-36.

38. Boniakowski AE, Kimball AS, Jacobs BN, et al. Macrophage-Mediated Inflammation in Normal and Diabetic Wound Healing. J Immunol 2017;199:17-24.

39. Mirza RE, Fang MM, Ennis WJ, et al. Blocking interleukin- $1 \beta$ induces a healing-associated wound macrophage phenotype and improves healing in type 2 diabetes. Diabetes 2013;62:2579-87.

40. Mast BA, Schultz GS. Interactions of cytokines, growth factors, and proteases in acute and chronic wounds. Wound Repair Regen 1996;4:411-20. 